Article

\title{
Existence and Stability Analysis for Fractional Impulsive Caputo Difference-Sum Equations with Periodic Boundary Condition
}

\author{
Rujira Ouncharoen ${ }^{1}$, Saowaluck Chasreechai ${ }^{2, *}$ and Thanin Sitthiwirattham ${ }^{3, *(D)}$
}

1 Research Center in Mathematics and Applied Mathematics, Department of Mathematics, Faculty of Science, Chiang Mai University, Chiang Mai 50200, Thailand; rujira.o@cmu.ac.th

2 Department of Mathematics, Faculty of Applied Science, King Mongkut's University of Technology

North Bangkok, Bangkok 10800, Thailand

3 Mathematics Department, Faculty of Science and Technology, Suan Dusit University,

Bangkok 10300, Thailand

* Correspondence: saowaluck.c@sci.kmutnb.ac.th (S.C.); thanin_sit@dusit.ac.th (T.S.)

Received: 6 May 2020; Accepted: 21 May 2020; Published: 22 May 2020

check for updates

\begin{abstract}
In this paper, by using the Banach contraction principle and the Schauder's fixed point theorem, we investigate existence results for a fractional impulsive sum-difference equations with periodic boundary conditions. Moreover, we also establish different kinds of Ulam stability for this problem. An example is also constructed to demonstrate the importance of these results.
\end{abstract}

Keywords: existence; impulse; Ulam-Hyers stability; fractional Caputo difference equations; boundary value problem

JEL Classification: 39A05; 39A12

\section{Introduction}

In this paper, we study the following periodic boundary value problem for fractional impulsive difference-sum equations:

$$
\begin{aligned}
& \Delta_{C}^{\alpha} u(t)=F\left[t+\alpha-1, u(t+\alpha-1), \Psi^{\gamma} u(t+\alpha-1)\right], \quad t \in \mathbb{N}_{0, T}, t+\alpha-1 \neq t_{k} \\
& A u(\alpha-1)+B \Delta^{-\beta} u(\alpha+\beta-1)=C u(T+\alpha)+D \Delta^{-\beta} u(T+\alpha+\beta)
\end{aligned}
$$

where the impluse conditions subjected to $I_{k}, J_{k}: \mathbb{R} \rightarrow \mathbb{R}$ are given by

$$
\begin{aligned}
& \Delta u\left(t_{k}\right)=I_{k}\left(u\left(t_{k}-1\right)\right), \quad k=1,2, \ldots, p \\
& \Delta\left(\Delta^{-\beta} u\left(t_{k}+\beta\right)\right)=J_{k}\left(\Delta^{-\beta} u\left(t_{k}+\beta-1\right)\right), k=1,2, \ldots, p
\end{aligned}
$$

and $\alpha, \beta \in(0,1) ; \mathbb{N}_{0, T}:=\{0,1, \ldots, T\} ; t_{k} \in \mathbb{N}_{\alpha, T+\alpha-1}$ and $t_{0}=\alpha-1<t_{1}<t_{2}<\ldots<t_{p}<T+$ $\alpha, t_{k+1}-t_{k} \geq 2 ; \Delta u\left(t_{k}\right)=u\left(t_{k}+1\right)-u\left(t_{k}\right) ; \Delta\left(\Delta^{-\beta} u\left(t_{k}+\beta\right)\right)=\Delta^{-\beta} u\left(t_{k}+\beta+1\right)-\Delta^{-\beta} u\left(t_{k}+\beta\right) ;$ $A, B, C, D \in \mathbb{R} ; F: \mathbb{N}_{\alpha-1, T+\alpha} \times \mathbb{R} \times \mathbb{R} \rightarrow \mathbb{R}$ is a continuous function ; and for $\varphi: \mathbb{N}_{\alpha-1, T+\alpha} \times$ $\mathbb{N}_{\alpha-1, T+\alpha} \rightarrow[0, \infty)$,

$$
\left(\Psi^{\gamma} u\right)(t):=\left[\Delta^{-\gamma} \varphi u\right](t+\gamma)=\frac{1}{\Gamma(\gamma)} \sum_{s=\alpha-\gamma-1}^{t-\gamma}(t-\sigma(s)) \frac{\gamma-1}{-1} \varphi(t, s+\gamma) u(s+\gamma)
$$


Fractional calculus has been gaining more attention over the past decade as this calculus has been addressed to various problems used in science and engineering; see [1-8]. For fractional difference calculus theory, which is the discrete case of fractional calculus, there is still not much interest among mathematical researchers. Basic knowledge of fractional difference calculus can be found in [9]. Some interesting results on fractional difference calculus have been studied, which has helped to develop the basic theory of this calculus; see [10-40] and references cited therein. The extension of applications of fractional difference calculus; see [41-43] and references cited therein.

Currently, the studies of boundary value problems for fractional difference equations are shown both extensive and more complex of conditions. There are some recent papers to study the existence and stability results of fractional difference equation [44-51]. However, a few paper has been made to develop the theory of the existence and stability results of fractional difference equations with impulse [52,53].

These results are the motivation for this research. By using the Banach contraction principle and the Schauder's fixed point theorem, we aim to prove the existence results for the problem (1) and (2). Moreover, we also provide a condition for the different kinds of Ulam stability for the problem (1) and (2). Then, we present an illustrative example.

\section{Preliminaries}

In this section, we recall some notations, definitions and lemmas which will be used in the main results.

Definition 1. The generalized falling function is defined as

$$
t^{\underline{\alpha}}:=\frac{\Gamma(t+1)}{\Gamma(t+1-\alpha)}
$$

when $t+1-\alpha$ is not a pole of the Gamma function. If $t+1$ is not a pole and when $t+1-\alpha$ is a pole, then $t^{\underline{\alpha}}=0$.

Definition 2. For $\alpha>0$ and $f$ defined on $\mathbb{N}_{a}:=\{a, a+1, \ldots\}$, the fractional sum of order $\alpha$ of $f$ is defined as

$$
\Delta^{-\alpha} f(t):=\frac{1}{\Gamma(\alpha)} \sum_{s=a}^{t-\alpha}(t-\sigma(s))^{\frac{\alpha-1}{-\alpha}} f(s),
$$

where $t \in \mathbb{N}_{a+\alpha}$ and $\sigma(s)=s+1$.

Definition 3. For $\alpha>0, N \in \mathbb{N}$ is satisfied with $\alpha \in(N-1, N]$ and $f$ defined on $\mathbb{N}_{a}$, the Riemann-Liouville fractional delta difference of order $\alpha$ of $f$ is defined as

$$
\Delta^{\alpha} f(t):=\Delta^{N} \Delta^{-(N-\alpha)} f(t)=\frac{1}{\Gamma(-\alpha)} \sum_{s=a}^{t+\alpha}(t-\sigma(s)) \frac{-\alpha-1}{f} f(s),
$$

where $t \in \mathbb{N}_{a+N-\alpha}$. The Caputo fractional difference of order $\alpha$ of $f$ is defined as

$$
\Delta_{C}^{\alpha} f(t):=\Delta^{-(N-\alpha)} \Delta^{N} f(t)=\frac{1}{\Gamma(N-\alpha)} \sum_{s=a}^{t-(N-\alpha)}(t-\sigma(s))^{\frac{N-\alpha-1}{N}} \Delta^{N} f(s),
$$

where $t \in \mathbb{N}_{a+N-\alpha}$. If $\alpha=N$, then $\Delta^{\alpha} f(t)=\Delta_{C}^{\alpha} f(t)=\Delta^{N} f(t)$.

Lemma 1 ([10]). Let $\alpha>0$ and $\alpha \in(N-1, N]$. Then

$$
\Delta^{-\alpha} \Delta_{C}^{\alpha} y(t)=y(t)+C_{0}+C_{1} t^{\underline{1}}+C_{2} t^{2}+\ldots+C_{N-1} t^{N-1},
$$


for some $C_{i} \in \mathbb{R}, 0 \leq i \leq N-1$.

Now, we aim to study the following linear variant of the boundary value problem (1) and (2).

Lemma 2. Let $\Lambda \neq 0 ; \alpha, \beta \in(0,1) ; A, B, C, D \in \mathbb{R} ; t_{0}=\alpha-1<t_{1}<t_{2}<\ldots<t_{p}<T+\alpha$; for $k=1,2, \ldots, p, t_{k+1}-t_{k} \geq 2$ and $t_{k} \in \mathbb{N}_{\alpha, T+\alpha-1} ; I_{k}, J_{k}: \mathbb{R} \rightarrow \mathbb{R}$ and $h: \mathbb{N}_{\alpha-1, T+\alpha} \rightarrow \mathbb{R}$ be continuous. Then the following problem

$$
\begin{aligned}
& \Delta_{C}^{\alpha} u(t)=h(t+\alpha-1), \quad t \in \mathbb{N}_{0, T}, t+\alpha-1 \neq t_{k} \\
& A u(\alpha-1)+B \Delta^{-\beta} u(\alpha+\beta-1)=C u(T+\alpha)+D \Delta^{-\beta} u(T+\alpha+\beta)
\end{aligned}
$$

where the impluse conditions subjected to $I_{k}, J_{k}$ are given by

$$
\begin{aligned}
& \Delta u\left(t_{k}\right)=I_{k}\left(u\left(t_{k}-1\right)\right), k=1,2, \ldots, p \\
& \Delta\left(\Delta^{-\beta} u\left(t_{k}+\beta\right)\right)=J_{k}\left(\Delta^{-\beta} u\left(t_{k}+\beta-1\right)\right), k=1,2, \ldots, p
\end{aligned}
$$

has the unique solution which is in a form

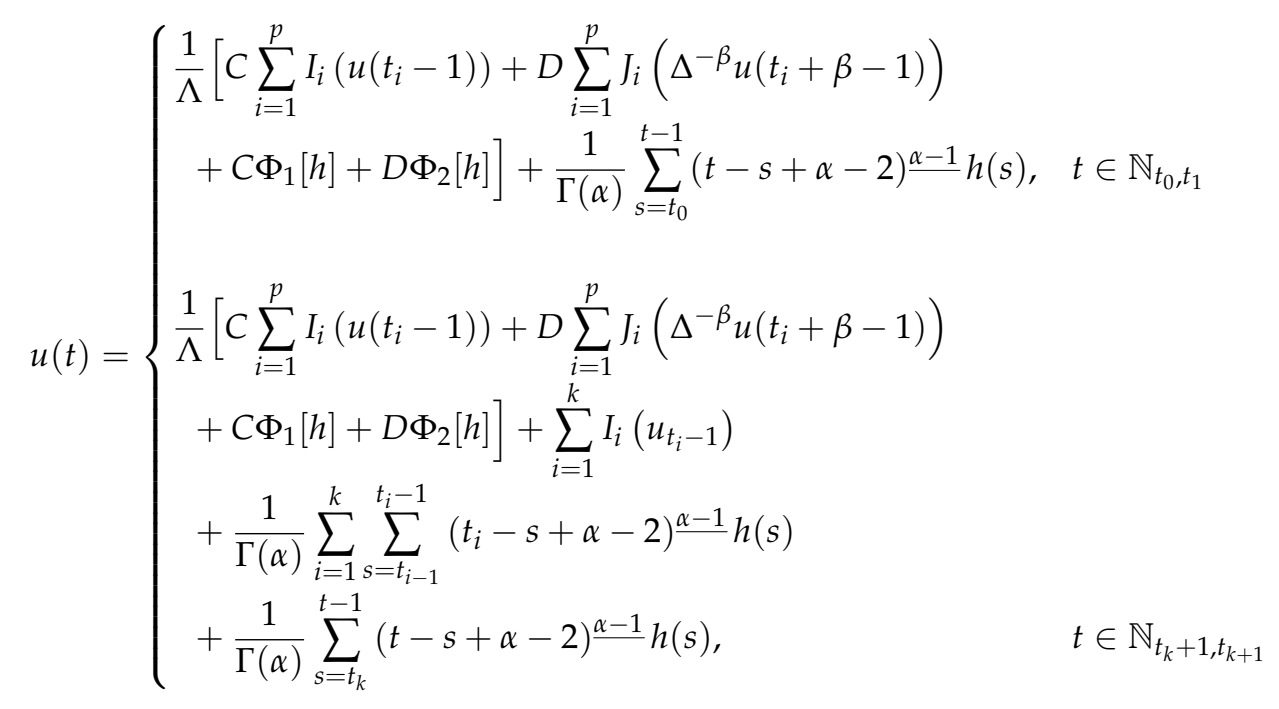

where $\Delta u\left(t_{k}\right)=u\left(t_{k}+1\right)-u\left(t_{k}\right) ; \Delta\left(\Delta^{-\beta} u\left(t_{k}+\beta\right)\right)=\Delta^{-\beta} u\left(t_{k}+\beta+1\right)-\Delta^{-\beta} u\left(t_{k}+\beta\right)$; the functionals $\Phi_{1}[h], \Phi_{2}[h]$ and the constant $\Lambda$ are defined by

$$
\begin{aligned}
\Phi_{1}[h]:= & \frac{1}{\Gamma(\alpha)}\left[\sum_{i=1}^{p} \sum_{s=t_{i-1}}^{t_{i}-1}\left(t_{i}-s+\alpha-2\right)^{\frac{\alpha-1}{}} h(s)+\sum_{s=t_{p}}^{T+\alpha-1}(T+2 \alpha-s-2)^{\frac{\alpha-1}{h}} h(s)\right] \\
\Phi_{2}[h]:= & \frac{1}{\Gamma(\alpha) \Gamma(\beta)}\left[\sum_{i=1}^{p} \sum_{r=t_{i-1}+1}^{t_{i}} \sum_{s=t_{i-1}}^{r-1}\left(t_{i}+\beta-s-1\right)^{\frac{\beta-1}{}}(r-s+\alpha-2)^{\frac{\alpha-1}{h}} h(s)\right. \\
& \left.+\sum_{s=t_{p}+1}^{T+\alpha} \sum_{s=t_{k}}^{r-1}(T+\alpha+\beta-s-1) \frac{\beta-1}{(}(r-s+\alpha-2)^{\frac{\alpha-1}{h}} h(s)\right] \\
\Lambda:= & A+B-C-\frac{D}{\Gamma(\beta)}\left[\sum_{i=1}^{p} \sum_{s=t_{i-1}}^{t_{i}}\left(t_{i}-s+\beta-1\right)^{\frac{\beta-1}{}}+\sum_{s=t_{p}}^{T+\alpha}(T+\alpha+\beta-s-1) \frac{\beta-1}{2}\right] .
\end{aligned}
$$


Proof. For $t \in \mathbb{N}_{t_{0}, t_{1}}$, by Lemma 1 and taking the fractional sum of order $\alpha$ for (3) and (4), a general solution can be written as

$$
\begin{aligned}
u(t) & =C_{0}+\frac{1}{\Gamma(\alpha)} \sum_{s=0}^{t-\alpha}(t-\sigma(s)) \frac{\alpha-1}{h} h(s+\alpha-1) \\
& =C_{0}+\frac{1}{\Gamma(\alpha)} \sum_{s=t_{0}}^{t-1}(t-s+\alpha-2) \frac{\alpha-1}{2} h(s)
\end{aligned}
$$

By substituting $t=t_{1}$ into (9), we have

$$
u\left(t_{1}\right)=C_{0}+\frac{1}{\Gamma(\alpha)} \sum_{s=t_{0}}^{t_{1}-1}\left(t_{1}-s+\alpha-2\right)^{\frac{\alpha-1}{2}} h(s) .
$$

If $t \in \mathbb{N}_{t_{1}+1, t_{2}}$, then we have

$$
\begin{aligned}
u(t)= & u\left(t_{1}+1\right)+\frac{1}{\Gamma(\alpha)} \sum_{s=t_{1}}^{t-1}(t-s+\alpha-2)^{\frac{\alpha-1}{}} h(s) \\
= & \Delta u\left(t_{1}\right)+u\left(t_{1}\right)+\frac{1}{\Gamma(\alpha)} \sum_{s=t_{1}}^{t-1}(t-s+\alpha-2)^{\frac{\alpha-1}{\alpha}} h(s) \\
= & I_{1}\left(u_{t_{1}}-1\right)+\left[C_{0}+\frac{1}{\Gamma(\alpha)} \sum_{s=t_{0}}^{t_{1}-1}\left(t_{1}-s+\alpha-2\right)^{\frac{\alpha-1}{}} h(s)\right] \\
& +\frac{1}{\Gamma(\alpha)} \sum_{s=t_{1}}^{t-1}(t-s+\alpha-2)^{\frac{\alpha-1}{h}} h(s) .
\end{aligned}
$$

If $t \in \mathbb{N}_{t_{2}+1, t_{3}}$, then we have

$$
\begin{aligned}
u(t)= & u\left(t_{2}+1\right)+\frac{1}{\Gamma(\alpha)} \sum_{s=t_{2}}^{t-1}(t-s+\alpha-2) \frac{\alpha-1}{h} h(s) \\
= & \Delta u\left(t_{2}\right)+u\left(t_{2}\right)+\frac{1}{\Gamma(\alpha)} \sum_{s=t_{2}}^{t-1}(t-s+\alpha-2)^{\frac{\alpha-1}{h}} h(s) \\
= & I_{2}\left(u_{t_{2}}-1\right)+\left[I_{1}\left(u_{t_{1}}-1\right)+C_{0}+\frac{1}{\Gamma(\alpha)} \sum_{s=t_{0}}^{t_{1}-1}\left(t_{1}-s+\alpha-2\right)^{\frac{\alpha-1}{}} h(s)\right. \\
& \left.+\frac{1}{\Gamma(\alpha)} \sum_{s=t_{1}}^{t_{2}-1}\left(t_{2}-s+\alpha-2\right)^{\frac{\alpha-1}{2}} h(s)\right]+\frac{1}{\Gamma(\alpha)} \sum_{s=t_{2}}^{t-1}(t-s+\alpha-2)^{\frac{\alpha-1}{2}} h(s) .
\end{aligned}
$$

Repeating the process, the solution $u(t)$ for $t \in \mathbb{N}_{t_{k}+1, t_{k+1}}(k=1,2, \ldots, p)$ can be written as

$$
\begin{aligned}
u(t)= & C_{0}+\sum_{i=1}^{k} I_{i}\left(u_{t_{i}}-1\right)+\frac{1}{\Gamma(\alpha)} \sum_{i=1}^{k} \sum_{s=t_{i-1}}^{t_{i}-1}\left(t_{i}-s+\alpha-2\right)^{\frac{\alpha-1}{}} h(s) \\
& +\frac{1}{\Gamma(\alpha)} \sum_{s=t_{k}}^{t-1}(t-s+\alpha-2)^{\frac{\alpha-1}{}} h(s) .
\end{aligned}
$$


Next, for $t \in \mathbb{N}_{t_{0}+\beta, t_{1}+\beta}$, taking the fractional sum of order $\beta$ for (9), we have

$$
\begin{aligned}
\Delta^{-\beta} u(t)= & \frac{C_{0}}{\Gamma(\beta)} \sum_{s=\alpha-1}^{t-\beta}(t-\sigma(s)) \frac{\beta-1}{} \\
& +\frac{1}{\Gamma(\alpha) \Gamma(\beta)} \sum_{s=\alpha-1}^{t-\beta} \sum_{s=0}^{r-\alpha}(t-\sigma(r)) \frac{\beta-1}{(r-\sigma(s)) \frac{\alpha-1}{h} h(s) .}
\end{aligned}
$$

Thus, for $t \in \mathbb{N}_{t_{0}, t_{1}}$, we have

$$
\begin{aligned}
\Delta^{-\beta} u(t+\beta)= & \frac{C_{0}}{\Gamma(\beta)} \sum_{s=t_{0}}^{t}(t+\beta-s-1) \frac{\beta-1}{} \\
& +\frac{1}{\Gamma(\alpha) \Gamma(\beta)} \sum_{s=t_{0}+1}^{t} \sum_{s=t_{0}}^{r-1}(t+\beta-r-1) \frac{\beta-1}{2}(r-s+\alpha-2) \frac{\alpha-1}{h} h(s) .
\end{aligned}
$$

By substituting $t=t_{1}$ into (15), we have

$$
\begin{aligned}
\Delta^{-\beta} u\left(t_{1}+\beta\right)= & \frac{C_{0}}{\Gamma(\beta)} \sum_{s=t_{0}}^{t_{1}}\left(t_{1}+\beta-s-1\right) \frac{\beta-1}{} \\
& +\frac{1}{\Gamma(\alpha) \Gamma(\beta)} \sum_{s=t_{0}+1}^{t_{1}} \sum_{s=t_{0}}^{r-1}\left(t_{1}+\beta-r-1\right) \frac{\beta-1}{\frac{\beta-1}{(}}(r-s+\alpha-2) \frac{\alpha-1}{h} h(s) .
\end{aligned}
$$

If $t \in \mathbb{N}_{t_{1}+1, t_{2}}$, then we have

$$
\begin{aligned}
& \Delta^{-\beta} u(t+\beta)=\Delta^{-\beta} u\left(t_{1}+\beta+1\right)+\frac{C_{0}}{\Gamma(\beta)} \sum_{s=t_{1}}^{t}(t+\beta-s-1) \frac{\beta-1}{\underline{\beta}} \\
& +\frac{1}{\Gamma(\alpha) \Gamma(\beta)} \sum_{s=t_{1}+1}^{t} \sum_{s=t_{1}}^{r-1}(t+\beta-r-1) \frac{\beta-1}{2}(r-s+\alpha-2)^{\frac{\alpha-1}{2}} h(s) \\
& =\Delta\left(\Delta^{-\beta} u\left(t_{1}+\beta\right)\right)+\Delta^{-\beta} u\left(t_{1}+\beta\right)+\frac{C_{0}}{\Gamma(\beta)} \sum_{s=t_{1}}^{t}(t+\beta-s-1) \underline{\beta-1}
\end{aligned}
$$

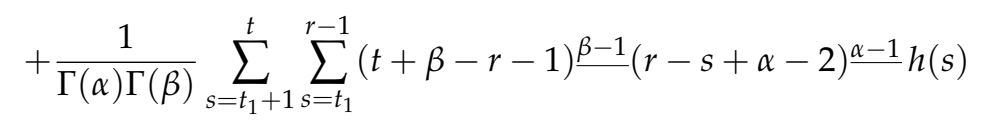

$$
\begin{aligned}
& =J_{1}\left(\Delta^{-\beta} u\left(t_{1}+\beta-1\right)\right)+\left[\frac{C_{0}}{\Gamma(\beta)} \sum_{s=t_{0}}^{t_{1}}\left(t_{1}+\beta-s-1\right) \frac{\beta-1}{\underline{\beta}}\right.
\end{aligned}
$$

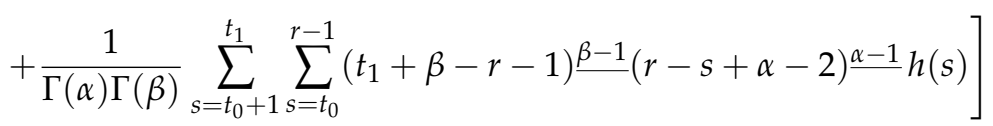

$$
\begin{aligned}
& +\frac{C_{0}}{\Gamma(\beta)} \sum_{s=t_{1}}^{t}(t+\beta-s-1) \frac{\beta-1}{}
\end{aligned}
$$

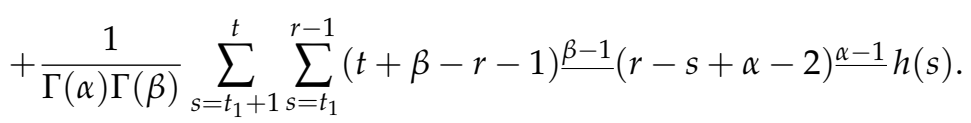


If $t \in \mathbb{N}_{t_{2}+1, t_{3}}$, then we have

$$
\begin{aligned}
& \Delta^{-\beta} u(t+\beta)=\Delta^{-\beta} u\left(t_{2}+\beta+1\right)+\frac{C_{0}}{\Gamma(\beta)} \sum_{s=t_{2}}^{t}(t+\beta-s-1) \underline{\beta-1} \\
& +\frac{1}{\Gamma(\alpha) \Gamma(\beta)} \sum_{s=t_{2}+1}^{t} \sum_{s=t_{2}}^{r-1}(t+\beta-r-1) \frac{\beta-1}{2}(r-s+\alpha-2) \frac{\alpha-1}{h} h(s) \\
& =\Delta\left(\Delta^{-\beta} u\left(t_{2}+\beta\right)\right)+\Delta^{-\beta} u\left(t_{2}+\beta\right)+\frac{C_{0}}{\Gamma(\beta)} \sum_{s=t_{2}}^{t}(t+\beta-s-1) \frac{\beta-1}{} \\
& +\frac{1}{\Gamma(\alpha) \Gamma(\beta)} \sum_{s=t_{2}+1}^{t} \sum_{s=t_{2}}^{r-1}(t+\beta-r-1) \frac{\beta-1}{2}(r-s+\alpha-2) \frac{\alpha-1}{h} h(s) \\
& =J_{1}\left(\Delta^{-\beta} u\left(t_{1}+\beta-1\right)\right)+J_{2}\left(\Delta^{-\beta} u\left(t_{2}+\beta-1\right)\right) \\
& +\frac{C_{0}}{\Gamma(\beta)}\left[\sum_{s=t_{0}}^{t_{1}}\left(t_{1}+\beta-s-1\right) \frac{\beta-1}{t_{2}}+\sum_{s=t_{1}}^{t_{2}}\left(t_{2}+\beta-s-1\right) \frac{\beta-1}{t_{1}}\right]
\end{aligned}
$$

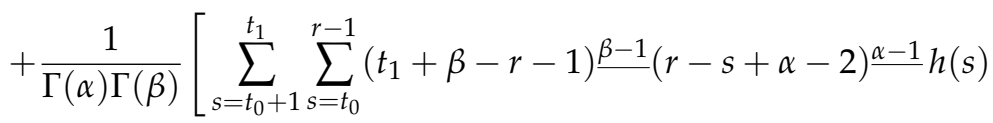

$$
\begin{aligned}
& \left.+\sum_{s=t_{1}+1}^{t_{2}} \sum_{s=t_{1}}^{r-1}\left(t_{2}+\beta-r-1\right) \frac{\beta-1}{(}(r-s+\alpha-2) \frac{\alpha-1}{c_{0}} h(s)\right] \\
& +\frac{C_{0}}{\Gamma(\beta)} \sum_{s=t_{2}}^{t}(t+\beta-s-1) \frac{\beta-1}{}
\end{aligned}
$$

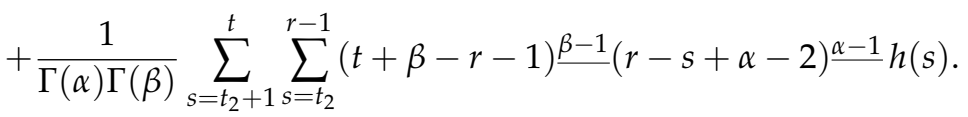

Repeating the process, the solution $u(t)$ for $t \in \mathbb{N}_{t_{k}+1, t_{k+1}}(k=1,2, \ldots, p)$ can be written as

$$
\begin{aligned}
& \Delta^{-\beta} u(t)=\sum_{i=1}^{k} J_{i}\left(\Delta^{-\beta} u_{t_{i}}+\beta-1\right) \\
& +\frac{C_{0}}{\Gamma(\beta)}\left[\sum_{i=1}^{k} \sum_{s=t_{i-1}}^{t_{i}-1}\left(t_{i}-s+\alpha-2\right) \frac{\beta-1}{h} h(s)+\sum_{s=t_{k}}^{t}(t-s+\beta-1) \frac{\beta-1}{]}\right]
\end{aligned}
$$

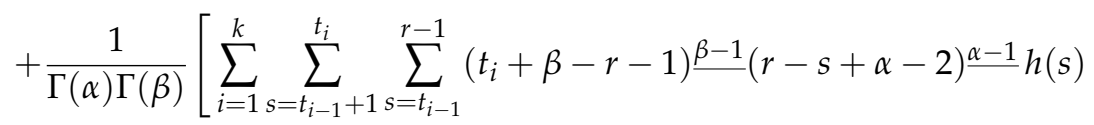

$$
\begin{aligned}
& +\sum_{s=t_{k}+1}^{t} \sum_{s=t_{k}}^{r-1}(t+\beta-r-1) \frac{\beta-1}{\left.(r-s+\alpha-2) \frac{\alpha-1}{2} h(s)\right] .}
\end{aligned}
$$

By substituting $t=\alpha-1$ into (9), (16); $t=T+\alpha$ into (13), (19); and using the condition $A u(\alpha-1)+$ $B \Delta^{-\beta} u(\alpha+\beta-1)=C u(T+\alpha)+D \Delta^{-\beta} u(T+\alpha+\beta)$, we have

$$
C_{0}=\frac{1}{\Lambda}\left[C \sum_{i=1}^{p} I_{i}\left(u\left(t_{i}-1\right)\right)+D \sum_{i=1}^{p} J_{i}\left(\Delta^{-\beta} u\left(t_{i}+\beta-1\right)\right)+C \Phi_{1}[h]+D \Phi_{2}[h]\right]
$$

where $\Phi_{1}[h], \Phi_{2}[h]$ and $\Lambda$ are defined in (6)-(8), respectively. Substituting $C_{0}$ into (9) and (13), we have (5). 


\section{Main Results}

\subsection{Existence and Uniqueness Solution}

Let $\mathcal{C}=C\left(\mathbb{N}_{\alpha-1, T+\alpha}, \mathbb{R}\right)$ be the Banach space equipped with the norm $\|u\|=\max _{t \in \mathbb{N}_{\alpha-1, T+\alpha}}|u(t)|$. Defined the operator $\mathcal{T}: \mathcal{C} \rightarrow \mathcal{C}$ by

$$
(\mathcal{T} u)(t):=\left\{\begin{aligned}
\frac{1}{\Lambda}\left[C \sum_{i=1}^{p} I_{i}\left(u\left(t_{i}-1\right)\right)+D \sum_{i=1}^{p} J_{i}\left(\Delta^{-\beta} u\left(t_{i}+\beta-1\right)\right)+C \Phi_{1}^{*}[F u]+D \Phi_{2}^{*}[F u]\right] \\
\quad+\frac{1}{\Gamma(\alpha)} \sum_{s=t_{0}}^{t-1}(t-s+\alpha-2) \frac{\alpha-1}{\Lambda} F\left[s, u(s), \Psi^{\gamma} u(s)\right], \quad t \in \mathbb{N}_{t_{0}, t_{1}} \\
\frac{1}{\Lambda}\left[C \sum_{i=1}^{p} I_{i}\left(u\left(t_{i}-1\right)\right)+D \sum_{i=1}^{p} J_{i}\left(\Delta^{-\beta} u\left(t_{i}+\beta-1\right)\right)\right. \\
\quad+C \Phi_{1}^{*}[F u]+D \Phi_{2}^{*}[F u]+\sum_{i=1}^{k} I_{i}\left(u_{t_{i}}-1\right) \\
\quad+\frac{1}{\Gamma(\alpha)} \sum_{i=1}^{k} \sum_{s=t_{i-1}}^{t_{i}-1}\left(t_{i}-s+\alpha-2\right)^{\frac{\alpha-1}{2}} F[s, u(s), \Psi \gamma u(s)] \\
\quad+\frac{1}{\Gamma(\alpha)} \sum_{s=t_{k}}^{t-1}(t-s+\alpha-2)^{\frac{\alpha-1}{2}} F\left[s, u(s), \Psi^{\gamma} u(s)\right],
\end{aligned}\right.
$$

where the functionals $\Phi_{1}^{*}[F u]$ and $\Phi_{2}^{*}[F u]$ are defined as

$$
\begin{aligned}
\Phi_{1}^{*}[F u]= & \frac{1}{\Gamma(\alpha)}\left[\sum_{i=1}^{p} \sum_{s=t_{i-1}}^{t_{i}-1}\left(t_{i}-s+\alpha-2\right)^{\frac{\alpha-1}{}} F\left[s, u(s), \Psi^{\gamma} u(s)\right]\right. \\
& \left.+\sum_{s=t_{p}}^{T+\alpha-1}(T+2 \alpha-s-2)^{\frac{\alpha-1}{}} F\left[s, u(s), \Psi^{\gamma} u(s)\right]\right] \\
\Phi_{2}^{*}[F u]= & \frac{1}{\Gamma(\alpha) \Gamma(\beta)}\left[\sum_{i=1}^{p} \sum_{r=t_{i-1}}^{t_{i}} \sum_{s=t_{i-1}}^{r-1}\left(t_{i}+\beta-s-1\right) \frac{\beta-1}{(r-s+\alpha-2) \frac{\alpha-1}{x} \times}\right. \\
& F\left[s, u(s), \Psi^{\gamma} u(s)\right]+\sum_{s=t_{p}+1}^{T+\alpha} \sum_{s=t_{k}}^{r-1}(T+\alpha+\beta-s-1) \frac{\beta-1}{(r-s+\alpha-2) \frac{\alpha-1}{x} \times} \\
& F\left[s, u(s), \Psi^{\gamma} u(s)\right]
\end{aligned}
$$

and constant $\Lambda$ is given in (8). Observe that the operator in (21) has the fixed points which are the solutions of the problem (1) and (2).

Theorem 1. Let $F: \mathbb{N}_{\alpha-1, T+\alpha} \times \mathbb{R} \times \mathbb{R} \rightarrow \mathbb{R}, I_{k}, J_{k}: \mathbb{R} \rightarrow \mathbb{R}, k=1,2, \ldots, p$ be continuous; $\varphi: \mathbb{N}_{\alpha-1, T+\alpha} \times$ $\mathbb{N}_{\alpha-1, T+\alpha} \rightarrow[0, \infty)$ be continuous with $\varphi_{0}=\max \left\{\varphi(t-1, s):(t, s) \in \mathbb{N}_{\alpha-1, T+\alpha} \times \mathbb{N}_{\alpha-1, T+\alpha}\right\}$. In addition, suppose that

$\left(H_{1}\right) \quad$ There exist constants $\ell_{1}, \ell_{2}>0$ such that

$$
\left|F\left[t, u(t),\left(\Psi^{\gamma} u\right)(t)\right]-F\left[t, v(t),\left(\Psi^{\gamma} v\right)(t)\right]\right| \leq \ell_{1}|u-v|+\ell_{2}\left|\left(\Psi^{\gamma} u\right)-\left(\Psi^{\gamma} v\right)\right|
$$

for each $t \in \mathbb{N}_{\alpha-1, \alpha+T}$ and $u, v \in \mathbb{R}$. 
$\left(H_{2}\right) \quad$ There exist constants $\lambda_{1}, \lambda_{2}>0$ such that

$$
\begin{aligned}
\left|I_{k}(u)-I_{k}(v)\right| & \leq \lambda_{1}|u-v| \\
\left|J_{k}\left(\Delta^{-\beta} u\right)-J_{k}\left(\Delta^{-\beta_{v}}\right)\right| & \leq \lambda_{2} \mid \Delta^{-\beta} u-\Delta^{-\beta_{v} \mid}
\end{aligned}
$$

for each $u, v \in \mathcal{C}$ and $k=1,2, \ldots, p$.

$\left(H_{3}\right) \quad \mathcal{L P}+\lambda_{1} \mathcal{Q}+\lambda_{2} \mathcal{R}<1$,

where

$$
\begin{aligned}
\mathcal{L} & =\ell_{1}+\ell_{2} \varphi_{0} \frac{(T+\alpha+\gamma) \underline{\gamma}}{\Gamma(\gamma+1)} \\
\mathcal{P} & =\left(\left|\frac{C}{\Lambda}\right|+1\right) \Omega_{1}+\left|\frac{D}{\Lambda}\right| \Omega_{2} \\
\mathcal{Q} & =\frac{p(p+1)}{2}\left(\left|\frac{C}{\Lambda}\right|+1\right) \\
\mathcal{R} & =\left|\frac{D}{\Lambda}\right| \frac{(T+\alpha+\beta)^{\underline{\beta}}}{\Gamma(\beta+1)} \\
\Omega_{1} & =\left[\frac{p(p+1)}{2}+1\right] \frac{(T+\alpha)^{\underline{\alpha}}}{\Gamma(\alpha+1)} \\
\Omega_{2} & =\left[\frac{p(p+1)}{2}+1\right] \frac{(T+\alpha)^{\underline{\alpha}}(T+\alpha+\beta)^{\underline{\beta}}}{\Gamma(\alpha+1) \Gamma(\beta+1)} .
\end{aligned}
$$

Therefore, the problem (1) and (2) has a unique solution on $\mathbb{N}_{\alpha-1, \alpha+T}$.

Proof. We will show that $\mathcal{T}$ is a contraction. Let

$$
\mathcal{H}|u-v|(t):=\left|F\left[t, u(t),\left(\Psi^{\gamma} u\right)(t)\right]-F\left[t, v(t),\left(\Psi^{\gamma} v\right)(t)\right]\right| .
$$

For any $u, v \in \mathcal{C}$, we have

$$
\begin{aligned}
& \left|\Phi_{1}^{*}[F u]-\Phi_{1}^{*}[F v]\right| \\
= & \frac{1}{\Gamma(\alpha)}\left|\sum_{i=1}^{p} \sum_{s=t_{i-1}}^{t_{i}-1}\left(t_{i}-s+\alpha-2\right)^{\frac{\alpha-1}{}} \mathcal{H}\right| u-v\left|(s)+\sum_{s=t_{p}}^{T+\alpha-1}(T+2 \alpha-s-2)^{\frac{\alpha-1}{}} \mathcal{H}\right| u-v|(s)| \\
\leq & \frac{\left[\ell_{1}|u-v|+\ell_{2}\left|\left(\Psi^{\gamma} u\right)-\left(\Psi_{\gamma} v\right)\right|\right]}{\Gamma(\alpha)}\left|\sum_{i=1}^{p} \sum_{s=t_{i-1}}^{t_{i}-1}\left(t_{i}-s+\alpha-2\right)^{\frac{\alpha-1}{}}+\sum_{s=t_{p}}^{T+\alpha-1}(T+2 \alpha-s-2)^{\frac{\alpha-1}{}}\right| \\
\leq & {\left[\ell_{1}+\ell_{2} \varphi_{0} \frac{(T+\alpha+\gamma) \underline{\gamma}}{\Gamma(\gamma+1)}\right]|u-v|\left[\frac{p(p+1)}{2}+1\right] \frac{(T+\alpha)^{\underline{\alpha}}}{\Gamma(\alpha+1)} } \\
= & \mathcal{L} \Omega_{1}|u-v| .
\end{aligned}
$$

Similary, we get

$$
\left|\Phi_{2}^{*}[F u]-\Phi_{2}^{*}[F v]\right| \leq \mathcal{L} \Omega_{2}|u-v| .
$$


Next, for each $t \in \mathbb{N}_{t_{k}+1, t_{k+1}}, k=1,2, \ldots, p$, we obtain

$$
\begin{aligned}
& |(\mathcal{T} u)(t)-(\mathcal{T} v)(t)| \\
& \leq \frac{1}{\Lambda}\left|C \sum_{i=1}^{p}\right| I_{i}\left(u_{t_{i}}-1\right)-I_{k}\left(v_{t_{i}}-1\right) \mid \\
& +D \sum_{i=1}^{p} \mid J_{i}\left(\Delta^{-\beta} u\left(t_{i}+\beta-1\right)\right)-J_{i}\left(\Delta^{\left.-\beta_{v}\left(t_{i}+\beta-1\right)\right) \mid}\right. \\
& +C\left|\Phi_{1}^{*}[F u]-\Phi_{1}^{*}[F v]\right|+D\left|\Phi_{2}^{*}[F u]-\Phi_{2}^{*}[F v]\right|\left|+\sum_{i=1}^{k}\right| I_{i}\left(u_{t_{i}}-1\right)-I_{k}\left(v_{t_{i}}-1\right) \mid \\
& +\frac{1}{\Gamma(\alpha)} \sum_{i=1}^{k} \sum_{s=t_{i-1}}^{t_{i}-1}\left(t_{i}-s+\alpha-2\right)^{\frac{\alpha-1}{}} \mathcal{H}|u-v|(s) \\
& +\frac{1}{\Gamma(\alpha)} \sum_{s=t_{k}}^{t-1}(t-s+\alpha-2)^{\frac{\alpha-1}{H}} \mathcal{H}|u-v|(s) \\
& \leq \frac{1}{|\Lambda|}\left[|C| \frac{p(p+1)}{2} \lambda_{1}|u-v|+|D| \frac{p(p+1)}{2} \lambda_{2}\left|\Delta^{-\beta} u-\Delta^{-\beta_{v}}\right|+|C| \mathcal{L} \Omega_{1}|u-v|\right. \\
& \left.+|D| \mathcal{L} \Omega_{2}|u-v|\right]+\frac{p(p+1)}{2} \lambda_{1}|u-v|+\left[\frac{p(p+1)}{2}+1\right] \frac{(T+\alpha)^{\underline{\alpha}}}{\Gamma(\alpha+1)} \mathcal{L}|u-v| \\
& \leq\left\{\frac{1}{|\Lambda|}\left[|C| \frac{p(p+1)}{2} \lambda_{1}+|C| \mathcal{L} \Omega_{1}+|D| \mathcal{L} \Omega_{2}\right]+\frac{p(p+1)}{2} \lambda_{1}+\left[\frac{p(p+1)}{2}+1\right]\right. \\
& \left.\frac{(T+\alpha)^{\underline{\alpha}}}{\Gamma(\alpha+1)} \mathcal{L}\right\}|u-v|+\left|\frac{D}{\Lambda}\right| \frac{p(p+1)}{2} \lambda_{2} \frac{(T+\alpha+\beta)^{\underline{\beta}}}{\Gamma(\beta+1)}|u-v| \\
& \leq\left[\mathcal{L P}+\lambda_{1} \mathcal{Q}+\lambda_{2} \mathcal{R}\right]|u-v| \text {. }
\end{aligned}
$$

Obviously, for each $t \in \mathbb{N}_{t_{0}, t_{1}}$, we obtain $|(\mathcal{T} u)(t)-(\mathcal{T} v)(t)|<|u-v|$.

Thus, for each $t \in \mathbb{N}_{\alpha-1, T+\alpha}$, we have $\|(\mathcal{T} u)(t)-(\mathcal{T} v)(t)\| \leq\|u-v\|$.

So, $\mathcal{T}$ is a contraction. By the Banach contraction principle, we can conclude that $\mathcal{T}$ has a fixed point. Hence, the problem (1) and (2) has a unique solution on $t \in \mathbb{N}_{\alpha-1, \alpha+T}$.

\subsection{Existence of at Least One Solution}

In the next result, we use of the Schauder's fixed point theorem to discuss the existence of at least one solution of (1) and (2).

Theorem 2. Suppose that $\left(H_{1}\right)$ and $\left(H_{3}\right)$ hold. Then, there exists at least one solution for the problem (1) and (2).

Proof. We organize the proof as follows:

Step I. Examine that $\mathcal{T}$ map bounded sets into bounded sets in $B_{R}=\{u \in \mathcal{C}:\|u\| \leq R\}$. Let $\max |F(t, 0,0,0)|=K, \max \left|I_{k}(u)\right|=M, \max \left|J_{k}\left(\Delta^{-\beta} u\right)\right|=M$, and choose a constant

$$
R \geq \frac{\mathcal{P} K}{1-\mathcal{L P}+M \mathcal{Q}+N \mathcal{R}} .
$$

Let

$$
|\mathcal{S}(t, u, 0)|=\left|F\left[t, u, \Delta^{-\beta} u\right]-F[t, 0,0]\right|+|F[t, 0,0]| .
$$


For any $u, v \in \mathcal{C}$, we obtain

$$
\begin{aligned}
& \left|\Phi_{1}^{*}[F u]\right| \\
= & \frac{1}{\Gamma(\alpha)}\left|\sum_{i=1}^{p} \sum_{s=t_{i-1}}^{t_{i}-1}\left(t_{i}-s+\alpha-2\right)^{\frac{\alpha-1}{-}}\right| \mathcal{S}(s, u, 0)\left|+\sum_{s=t_{p}}^{T+\alpha-1}(T+2 \alpha-s-2)^{\frac{\alpha-1}{2}}\right| \mathcal{S}(s, u, 0)|| \\
\leq & \left(\left[\ell_{1}+\ell_{2} \varphi_{0} \frac{(T+\alpha+\gamma) \underline{\gamma}}{\Gamma(\gamma+1)}\right]|u|+K\right) \Omega_{1} \\
= & \mathcal{L} \Omega_{1} R+K \Omega_{1} .
\end{aligned}
$$

Similarly, we obtain

$$
\left|\Phi_{2}^{*}[F u]\right| \leq \mathcal{L} \Omega_{2} R+K \Omega_{2}
$$

So, for each $t \in \mathbb{N}_{t_{k}+1, t_{k+1}}, k=1,2, \ldots, p$, we get

$$
|(\mathcal{T} u)(t)| \leq[\mathcal{L P}+M \mathcal{Q}+N \mathcal{R}] R+K \mathcal{P} \leq R .
$$

Obviously, for each $t \in \mathbb{N}_{t_{0}, t_{1}}$, we have $|\mathcal{T} u(t)|<R$.

Therefore, $\|\mathcal{T} u\| \leq R$ for each $t \in \mathbb{N}_{\alpha-1, T+\alpha}$, which implies that $\mathcal{F}$ is uniformly bounded.

Step II. It is obvious that the operator $\mathcal{T}$ is continuous on $B_{R}$ since the continuity of $F$.

Step III. Examine that $\mathcal{T}$ is equicontinuous on $B_{R}$. For any $\epsilon>0$, there exists $\delta>0$ such that for $\tau_{1}, \tau_{2} \in \mathbb{N}_{\alpha-1, T+\alpha}$ with $\tau_{1}<\tau_{2}$

$$
\left|\tau_{2}^{\alpha}-\tau_{1}^{\alpha}\right|<\frac{\epsilon \Gamma(\alpha+1)}{\|F\|} \text { whenever }\left|\tau_{2}-\tau_{1}\right|<\delta .
$$

Then, we obtain

$$
\begin{aligned}
\left|(\mathcal{T} u)\left(\tau_{2}\right)-(\mathcal{T} u)\left(\tau_{1}\right)\right| \leq & \frac{\|F\|\left(T-\omega_{0}\right)^{\alpha}}{\Gamma_{q}(\alpha+1) \Gamma_{q}(\beta+1)} \mid \frac{1}{\Gamma(\alpha)} \sum_{s=t_{k}}^{\tau_{2}-1}\left(\tau_{2}-s+\alpha-2\right)^{\frac{\alpha-1}{\alpha}} \\
& -\frac{1}{\Gamma(\alpha)} \sum_{s=t_{k}}^{\tau_{1}-1}\left(\tau_{1}-s+\alpha-2\right)^{\frac{\alpha-1}{}} \mid \\
= & \frac{\|F\|}{\Gamma(\alpha+1)}\left|\tau_{2}^{\alpha}-\tau_{1}^{\alpha}\right|<\epsilon .
\end{aligned}
$$

This implies that the set $\mathcal{T}\left(B_{R}\right)$ is an equicontinuous set.

Hence, by the Arzelá-Ascoli theorem, we deduce that $\mathcal{T}: \mathcal{C} \rightarrow \mathcal{C}$ is completely continuous. Therefore, it follows from the Schauder's fixed point theorem that problem (1)-(2) has at least one solution.

\section{Ulam Stability Analysis Results}

Based on the concept in Wang et al. [54], we provide Ulam's type stability concepts for the problem (1) and (2). Consider the inequalities:

$$
\left\{\begin{array}{l}
\left|\Delta_{C}^{\alpha} z(t)-F\left[t+\alpha-1, z(t+\alpha-1), \Psi^{\gamma} z(t+\alpha-1)\right]\right| \leq \epsilon \\
\left|\Delta u\left(t_{k}\right)-I_{k}\left(u\left(t_{k}-1\right)\right)\right| \leq \epsilon \\
\left|\Delta\left(\Delta^{-\beta} u\left(t_{k}+\beta\right)\right)-J_{k}\left(\Delta^{-\beta} u\left(t_{k}+\beta-1\right)\right)\right| \leq \epsilon,
\end{array}\right.
$$




$$
\left\{\begin{array}{l}
\left|\Delta_{C}^{\alpha} z(t)-F\left[t+\alpha-1, z(t+\alpha-1), \Psi^{\gamma} z(t+\alpha-1)\right]\right| \leq \epsilon \rho(t) \\
\left|\Delta u\left(t_{k}\right)-I_{k}\left(u\left(t_{k}-1\right)\right)\right| \leq \epsilon \psi_{1} \\
\left|\Delta\left(\Delta^{-\beta} u\left(t_{k}+\beta\right)\right)-J_{k}\left(\Delta^{-\beta} u\left(t_{k}+\beta-1\right)\right)\right| \leq \epsilon \psi_{2}
\end{array}\right.
$$

for $t \in \mathbb{N}_{0, T}, t+\alpha-1 \neq t_{k}, k=1,2, \ldots, p$.

Definition 4. The problem (1) and (2) is the Ulam-Hyers stable if there exists a real number $c_{F, p}>0$ such that for each $\epsilon>0$ and for each solution $z \in \mathcal{C}$ of the inequality (38), there exists a solution $u \in \mathcal{C}$ of problem (1) and (2) with

$$
|z(t)-u(t)| \leq c_{F, p} \epsilon .
$$

The problem (1) and (2) is the generalized Ulam-Hyers stable, if we substitute the function $\theta_{F, p}(\epsilon) \in$ $C\left(\mathbb{R}^{+}, \mathbb{R}^{+}\right), \theta_{F, p}(0)=0$ for the constant $c_{F, p} \in$ on inequality (40).

Definition 5. The problem (1) and (2) is the Ulam-Hyers-Rassias stable with respect to $\left(\rho, \psi_{1}, \psi_{2}\right)$ if there exists a real number $c_{F, p, \rho}>0$ such that for each $\epsilon, \psi_{1}, \psi_{2}>0$ and for each solution $z \in \mathcal{C}$ of the inequality (40), there exists a solution $u \in \mathcal{C}$ of problem (1) and (2) with

$$
|z(t)-u(t)| \leq c_{F, p, \rho} \epsilon\left(\rho(t)+\psi_{1}+\psi_{2}\right) .
$$

The problem (1) and (2) is the generalized Ulam-Hyers-Rassias stable, if we substitute the function $\rho^{*}(t)$ for the function $\epsilon \rho(t)$ and the constants $\psi_{1}^{*}, \psi_{2}^{*}$ for on the constants $\epsilon \psi_{1}, \epsilon \psi_{2}$ on inequalities (39) and (41).

Remark 1. A function $z \in \mathcal{C}$ is a solution of the inequality (38) if and only if there exist $\phi \in \mathcal{C}$ (depend on $z$ ) and sequence $\omega_{k}, \kappa_{k}, k=1,2, \ldots, p$ with $\omega=\max \left\{\omega_{k}\right\}, \kappa=\max \left\{\kappa_{k}\right\}$, such that

(i) $|\phi(t)| \leq \epsilon$ for $t \in \mathbb{N}_{t_{k}+1, t_{k+1}}$ and $|\omega|,|\kappa| \leq \epsilon ;$

(ii) $\quad \Delta_{C}^{\alpha} z(t)=F\left[t+\alpha-1, z(t+\alpha-1), \Psi^{\gamma} z(t+\alpha-1)\right]+\phi(t+\alpha-1)$

for $t \in \mathbb{N}_{0, T}, t+\alpha-1 \neq t_{k}$;

(iii) $\quad \Delta u\left(t_{k}\right)=I_{k}\left(u\left(t_{k}-1\right)\right)+\omega_{k}$;

(iv) $\Delta\left(\Delta^{-\beta} u\left(t_{k}+\beta\right)\right)=J_{k}\left(\Delta^{-\beta} u\left(t_{k}+\beta-1\right)\right)+\kappa_{k}$.

Remark 2. A function $z \in \mathcal{C}$ is a solution of the inequality (39) if and only if there exist $\phi \in \mathcal{C}$ (depend on $z$ ) and sequence $\omega_{k}, \kappa_{k}, k=1,2, \ldots, p$ with $\omega=\max \left\{\omega_{k}\right\}, \kappa=\max \left\{\kappa_{k}\right\}$,

(i) $|\phi(t)| \leq \epsilon \rho(t)$ for $t \in \mathbb{N}_{t_{k}+1, t_{k+1},}|\omega| \leq \epsilon \psi_{1}$ and $|\kappa| \leq \epsilon \psi_{2} ;$

(ii) $\quad \Delta_{C}^{\alpha} z(t)=F\left[t+\alpha-1, z(t+\alpha-1), \Psi^{\gamma} z(t+\alpha-1)\right]+\phi(t+\alpha-1)$

for $t \in \mathbb{N}_{0, T}, t+\alpha-1 \neq t_{k}$;

(iii) $\quad \Delta u\left(t_{k}\right)=I_{k}\left(u\left(t_{k}-1\right)\right)+\omega_{k}$;

(iv) $\Delta\left(\Delta^{-\beta} u\left(t_{k}+\beta\right)\right)=J_{k}\left(\Delta^{-\beta} u\left(t_{k}+\beta-1\right)\right)+\kappa_{k}$.

Lemma 3. If $z \in \mathcal{C}$ is a solution of the inequality (38), then for $\epsilon>0, z$ is solution of the inequality

$$
|z(t)-(\mathcal{T} z)(t)| \leq \epsilon(\mathcal{P}+\mathcal{Q}+\mathcal{R}),
$$

where $\mathcal{P}, \mathcal{Q}$ and $\mathcal{R}$ are defined in (25)-(27). 
Proof. From Remark 1 and Lemma 2, we have

$$
\begin{aligned}
z(t)= & \frac{1}{\Lambda}\left[C \sum_{i=1}^{p}\left[I_{i}\left(u\left(t_{i}-1\right)\right)+\omega_{i}\right]+D \sum_{i=1}^{p}\left[J_{i}\left(\Delta^{-\beta} u\left(t_{i}+\beta-1\right)\right)+\kappa_{i}\right]\right. \\
& \left.+C \Phi_{1}^{*}[F u+\phi]+D \Phi_{2}^{*}[F u+\phi]\right]+\sum_{i=1}^{k}\left[I_{i}\left(u_{t_{i}}-1\right)+\omega_{i}\right] \\
& +\frac{1}{\Gamma(\alpha)} \sum_{i=1}^{k} \sum_{s=t_{i-1}}^{t_{i}-1}\left(t_{i}-s+\alpha-2\right)^{\frac{\alpha-1}{}}\left[F\left[s, u(s), \Psi^{\gamma} u(s)\right]+\phi(s)\right] \\
& +\frac{1}{\Gamma(\alpha)} \sum_{s=t_{k}}^{t-1}(t-s+\alpha-2)^{\frac{\alpha-1}{2}}\left[F\left[s, u(s), \Psi^{\gamma} u(s)\right]+\phi(s)\right] .
\end{aligned}
$$

Hence, we obtain

$$
\begin{aligned}
|z(t)-(\mathcal{T} z)(t)| \leq & \frac{1}{|\Lambda|}\left[|C| \sum_{i=1}^{p}\left|\omega_{i}\right|+|D| \sum_{i=1}^{p}\left|\kappa_{i}\right|+|C| \Phi_{1}^{*}[|\phi|]+D \Phi_{2}^{*}[|\phi|]\right] \\
& +\sum_{i=1}^{k}\left|\omega_{i}\right|+\frac{1}{\Gamma(\alpha)} \sum_{i=1}^{k} \sum_{s=t_{i-1}}^{t_{i}-1}\left(t_{i}-s+\alpha-2\right)^{\frac{\alpha-1}{}}|\phi(s)| \\
& +\frac{1}{\Gamma(\alpha)} \sum_{s=t_{k}}^{t-1}(t-s+\alpha-2)^{\frac{\alpha-1}{}}|\phi(s)| \\
\leq & \frac{p(p+1)}{2}\left[\left(\left|\frac{C}{\Lambda}\right|+1\right)|\omega|+\left|\frac{D}{\Lambda}\right||\kappa|\right]+\frac{\|\phi\|}{|\Lambda|}\left[|C| \Omega_{1}+|D| \Omega_{2}\right] \\
& +\frac{\|\phi\|}{\Gamma(\alpha+1)}\left[\frac{p(p+1)}{2}(T+\alpha)^{\underline{\alpha}}+t^{\underline{\alpha}}\right] \\
\leq & \|\phi\| \mathcal{P}+|\omega| \mathcal{Q}+|\kappa| \mathcal{R} \\
\leq & \epsilon(\mathcal{P}+\mathcal{Q}+\mathcal{R})
\end{aligned}
$$

where $\mathcal{P}, \mathcal{Q}, \mathcal{R}, \Omega_{1}, \Omega_{2}$ are defined in (25)-(29). This completes the proof.

Theorem 3. Suppose that $\left(H_{1}\right)-\left(H_{3}\right)$ hold with $1-\left(\mathcal{L P}+\lambda_{1} \mathcal{Q}+\lambda_{2} \mathcal{R}\right) \neq 0$. Then, problem (1) and (2) is Ulam-Hyers stable.

Proof. Suppose $z \in \mathcal{C}$ is a solution of (38) and assume that $x(t)$ is the unique solution of problem (1) and (2). Consider

$$
\begin{aligned}
|z(t)-x(t)| & =|z(t)-(\mathcal{T} z)(t)+(\mathcal{T} z)(t)-x(t)| \\
& \leq|z(t)-(\mathcal{T} z)(t)|+|(\mathcal{T} z)(t)-x(t)| \\
& \leq \epsilon(\mathcal{P}+\mathcal{Q}+\mathcal{R})+\left(\mathcal{L} \mathcal{P}+\lambda_{1} \mathcal{Q}+\lambda_{2} \mathcal{R}\right)|z(t)-x(t)|
\end{aligned}
$$

from which we obtain

$$
|z(t)-x(t)| \leq \frac{\epsilon(\mathcal{P}+\mathcal{Q}+\mathcal{R})}{\left|1-\left(\mathcal{L P}+\lambda_{1} \mathcal{Q}+\lambda_{2} \mathcal{R}\right)\right|}
$$

By setting $c_{F, p}=\frac{(\mathcal{P}+\mathcal{Q}+\mathcal{R})}{\left|1-\left(\mathcal{L P}+\lambda_{1} \mathcal{Q}+\lambda_{2} \mathcal{R}\right)\right|}$, we have

$$
|z(t)-x(t)| \leq c_{F, p} \epsilon
$$


Hence, the problem (1) and (2) is Ulam-Hyers stable. Next, by setting $\theta_{F, p}(\epsilon)=c_{F, p} \epsilon$ with $\theta_{F, p}(0)=0$, the problem (1) and (2) is generalized Ulam-Hyers stable.

Lemma 4. If $z \in \mathcal{C}$ is a solution of the inequality (39) and assume that

$\left(H_{4}\right) \quad \frac{p(p+1)}{2}\left[\left(\left|\frac{C}{\Lambda}\right|+1\right)|\omega|+\left|\frac{D}{\Lambda}\right||\kappa|\right]+\frac{\|\phi\|}{|\Lambda|}\left[|C| \Omega_{1}+|D| \Omega_{2}\right]+\frac{\|\phi\|}{\Gamma(\alpha+1)}\left[\frac{p(p+1)}{2}(T+\alpha)^{\underline{\alpha}}+t^{\underline{\alpha}}\right]$ $\leq \epsilon \mathcal{O}\left[\rho(t)+\psi_{1}+\psi_{2}\right]$,

then for $\epsilon>0, z$ is solution of the following inequality

$$
|z(t)-(\mathcal{T} z)(t)| \leq \epsilon \mathcal{O}\left[\rho(t)+\psi_{1}+\psi_{2}\right]
$$

where $\mathcal{O}=\max \{\mathcal{P}, \mathcal{Q}, \mathcal{R}\}$ and $\mathcal{P}, \mathcal{Q}, \mathcal{R}, \Omega_{1}, \Omega_{2}$ are defined in (25)-(29).

Proof. By the same argument of the proof Lemma 3, we have

$$
\begin{aligned}
|z(t)-(\mathcal{T} z)(t)| \leq & \frac{p(p+1)}{2}\left[\left(\left|\frac{C}{\Lambda}\right|+1\right)|\omega|+\left|\frac{D}{\Lambda}\right||\kappa|\right]+\frac{\|\phi\|}{|\Lambda|}\left[|C| \Omega_{1}+|D| \Omega_{2}\right] \\
& +\frac{\|\phi\|}{\Gamma(\alpha+1)}\left[\frac{p(p+1)}{2}(T+\alpha)^{\underline{\alpha}}+t^{\underline{\alpha}}\right] \\
\leq & \epsilon\left[\rho(t) \mathcal{P}+\psi_{1} \mathcal{Q}+\psi_{2} \mathcal{R}\right] \\
\leq & \epsilon \mathcal{O}\left[\rho(t)+\psi_{1}+\psi_{2}\right] .
\end{aligned}
$$

This completes the proof.

Theorem 4. Suppose that $\left(H_{1}\right)-\left(H_{3}\right)$ hold with $1-\left(\mathcal{L P}+\lambda_{1} \mathcal{Q}+\lambda_{2} \mathcal{R}\right) \neq 0$. Then, problem (1) and (2) is Ulam-Hyers-Rassias stable.

Proof. Suppose $z \in \mathcal{C}$ is a solution of (39) and assume that $x(t)$ is the unique solution of problem (1) and (2). Consider

$$
\begin{aligned}
|z(t)-x(t)| & =|z(t)-(\mathcal{T} z)(t)+(\mathcal{T} z)(t)-x(t)| \\
& \leq|z(t)-(\mathcal{T} z)(t)|+|(\mathcal{T} z)(t)-x(t)| \\
& \leq \epsilon \mathcal{O}\left(\rho(t)+\psi_{1}+\psi_{2}\right)+\left(\mathcal{L P}+\lambda_{1} \mathcal{Q}+\lambda_{2} \mathcal{R}\right)|z(t)-x(t)|
\end{aligned}
$$

from which we obtain

$$
|z(t)-x(t)| \leq \frac{\epsilon \mathcal{O}\left[\rho(t)+\psi_{1}+\psi_{2}\right]}{\left|1-\left(\mathcal{L P}+\lambda_{1} \mathcal{Q}+\lambda_{2} \mathcal{R}\right)\right|}
$$

By setting $c_{F, p}=\frac{\mathcal{O}}{\left|1-\left(\mathcal{L P}+\lambda_{1} \mathcal{Q}+\lambda_{2} \mathcal{R}\right)\right|}$, we have

$$
|z(t)-x(t)| \leq c_{F, p} \epsilon\left[\rho(t)+\psi_{1}+\psi_{2}\right] .
$$

Hence, the problem (1) and (2) is Ulam-Hyers-Rassias stable. Next, by setting $\rho^{*}(t)+\psi_{1}^{*}+\psi_{2}^{*}=$ $\epsilon\left[\rho(t)+\psi_{1}+\psi_{2}\right]$, the problem (1) and (2) is generalized Ulam-Hyers-Rassias stable. 


\section{An Example}

To show the application of our theorems, we provide the fractional impulsive difference-sum equations with periodic boundary conditions of the form

$$
\begin{aligned}
& \Delta_{C}^{\frac{1}{2}} u(t)=\frac{\cos ^{2}\left(t-\frac{1}{2}\right) \pi}{2\left(t-\frac{199}{2}\right)^{2}}\left[\frac{u^{2}+|u|}{|u|+1}+e^{-\sin ^{2}\left(t-\frac{1}{2}\right) \pi} \Psi \frac{1}{3} u\left(t-\frac{1}{2}\right)\right], t \in \mathbb{N}_{0, T}, t-\frac{1}{2} \neq t_{k} \\
& 10 u\left(\frac{1}{2}\right)+20 \Delta^{-\frac{2}{3}} u\left(\frac{1}{6}\right)=5 u\left(\frac{21}{2}\right)+15 \Delta^{-\frac{2}{3}} u\left(\frac{67}{6}\right)
\end{aligned}
$$

where the impluse conditions subjected to $I_{k}, J_{k}: \mathbb{R} \rightarrow \mathbb{R}$ are given by

$$
\begin{aligned}
& \Delta u\left(t_{k}\right)=\frac{1}{k+100} \cos \left|u\left(t_{k}-1\right)\right|, k=1,2,3 \\
& \Delta\left(\Delta^{-\frac{2}{3}} u\left(t_{k}+\frac{2}{3}\right)\right)=\frac{1}{(k+50)^{2}} \tan ^{-1}\left|\Delta^{-\frac{2}{3}} u\left(t_{k}-\frac{1}{3}\right)\right|, \quad k=1,2,3
\end{aligned}
$$

and $\varphi(t, s)=\frac{e^{-|s-t|}}{(t+10)^{3}}$ and $t_{k}=\frac{1}{2}+2 k\left(t_{1}=\frac{5}{2}, t_{2}=\frac{9}{2}, t_{3}=\frac{13}{2}\right)$.

Here $\alpha=\frac{1}{2}, \beta=\frac{2}{3}, \gamma=\frac{1}{3}, T=10, p=3, A=10, B=20, C=5, D=15$, and $F\left[t, u(t), \Psi^{\gamma} u(t)\right]=\frac{\cos ^{2} \pi t}{2(t+100)^{2}}\left[\frac{u^{2}+|u|}{|u|+1}+e^{-\sin ^{2} \pi t} \Psi^{\frac{1}{3}} u(t)\right]$.

We can find that

$$
\begin{gathered}
\Theta=6.74071,|\Lambda|=75.57065, \Omega_{1}=25.90098, \Omega_{2}=144.78000, \\
\mathcal{P}=46.7733, \mathcal{Q}=7.46312 \text { and } \mathcal{R}=0.73969 .
\end{gathered}
$$

Let $t \in \mathbb{N}_{\frac{1}{2}, \frac{1}{2}}$ and $u, v \in \mathbb{R}$, we have

$$
\left|F\left[t, u, \Psi^{\gamma} u\right]-F\left[t, v, \Psi^{\gamma} v\right]\right| \leq \frac{1}{2\left(\frac{1}{2}+100\right)^{2}}|u-v|+\frac{1}{2 e^{\pi}\left(\frac{1}{2}+100\right)^{2}}\left|\Psi^{\gamma} u-\Psi^{\gamma} v\right|
$$

So, $\left(H_{1}\right)$ holds with $\ell_{1}=0.000495, \ell_{2}=5.6095 \times 10^{-6}$, and we have $\varphi_{0}=0.00907$.

For all $u, v \in \mathcal{C}$ and $k=1,2,3$

$$
I_{k}(u)-I_{k}(v) \leq \frac{1}{101}|u-v| \text { and } J_{k}\left(\Delta^{-\beta} u\right)-I_{k}\left(\Delta^{-\beta_{v}}\right) \leq \frac{1}{51^{2}}\left|\Delta^{-\beta} u-\Delta^{-\beta_{v}}\right|
$$

So, $\left(H_{2}\right)$ holds with $\lambda_{1}=\frac{1}{101}, \lambda_{2}=\frac{1}{51^{2}}$.

Finally, $\left(H_{3}\right)$ holds with

$$
\mathcal{L P}+\lambda_{1} \mathcal{Q}+\lambda_{2} \mathcal{R} \approx 0.07418<1 .
$$

Hence, by Theorem 1, the boundary value problem (52) and (53) has a unique solution.

In view of Theorem 3, we have

$$
c_{F, p}=\frac{\mathcal{P}+\mathcal{Q}+\mathcal{R}}{\left|1-\left(\mathcal{L P}+\lambda_{1} \mathcal{Q}+\lambda_{2} \mathcal{R}\right)\right|} \approx 2128.38328
$$

Therefore, problem (52) and (53) is Ulam-Hyers stable and hence generalized Ulam-Hyers stable. 
By setting

$$
\begin{gathered}
\mathcal{c}_{F, p, \rho}=\frac{\mathcal{O}}{\left|1-\left(\mathcal{L} \mathcal{P}+\lambda_{1} \mathcal{Q}+\lambda_{2} \mathcal{R}\right)\right|} \approx 44.03976, \text { and } \\
\rho(t)=0.02412 t^{\underline{\alpha}}+1.12568, \psi_{1}=0.13677, \psi_{2}=0.01698 .
\end{gathered}
$$

Thus, by Theorem 4, problem (52) and (53) is Ulam-Hyers-Rassias stable and also generalized Ulam-Hyers-Rassias stable.

Author Contributions: Conceptualization, R.O. and S.C.; Formal analysis, R.O. and S.C.; Funding acquisition, S.C.; Investigation, R.O.; Methodology, R.O., S.C. and T.S.; Writing-original draft, R.O., S.C. and T.S.; Writing-review and editing, R.O., S.C. and T.S. All authors have read and agreed to the published version of the manuscript.

Funding: This research was funded by King Mongkut's University of Technology North Bangkok. Contract no.KMUTNB-61-GOV-D-65.

Acknowledgments: This research was supported by Chiang Mai University.

Conflicts of Interest: The authors declare no conflicts of interest regarding the publication of this paper.

\section{References}

1. Zhang, Y.; Liu, X.; Belić, M.R.; Zhong, W.; Zhang, Y.; Xiao, M. Propagation Dynamics of a Light Beam in a Fractional Schrödinger Equation. Phys. Rev. Lett. 2015, 115, 180403. [CrossRef] [PubMed]

2. Zingales, M.; Failla, G. The finite element method for fractional non-local thermal energy transfer in non-homogeneous rigid conductors. Commun. Nonlinear Sci. Numer. Simul. 2015, 29, 116-127. [CrossRef]

3. Lazopoulos, K.A.; Lazopoulos, A.K. On fractional bending of beams. Arch. Appl. Mech. 2016, 86, 1133-1145. [CrossRef]

4. Sumelka, W.; Voyiadjis, G.Z. A hyperelastic fractional damage material model with memory. Int. J. Solids Struct. 2017, 124, 151-160 [CrossRef]

5. Voyiadjis, G.Z.; Sumelka, W. Brain modelling in the framework of anisotropic hyperelasticity with time fractional damage evolution governed by the Caputo-Almeida fractional derivative. J. Mech. Behav. Biomed. 2019, 89, 209-216. [CrossRef] [PubMed]

6. Caputo, M.; Ciarletta, M.; Fabrizio, M.; Tibullo, V. Melting and solidification of pure metals by a phase-field model. Rend Lincei-Mat. Appl. 2017, 28, 463-478. [CrossRef]

7. Gómez-Aguilar, J.F. Fractional Meissner-Ochsenfeld effect in superconductors. Phys. Lett. B 2019, 33, 1950316. [CrossRef]

8. Rahimi, Z.; Rezazadeh, G.; Sumelka, W.; Yang, X.-J. A study of critical point instability of micro and nano beams under a distributed variable-pressure force in the framework of the inhomogeneous non-linear nonlocal theory. Arch. Mech. 2017, 69, 413-433.

9. Goodrich, C.S.; Peterson, A.C. Discrete Fractional Calculus; Springer: New York, NY, USA, 2015.

10. Abdeljawad, T. On Riemann and Caputo fractional differences. Comput. Math. Appl. 2011, 62, 1602-1611. [CrossRef]

11. Abdeljawad, T. Dual identities in fractional difference calculus within Riemann. Adv. Differ. Equ. 2013, 2013, 36. [CrossRef]

12. Abdeljawad, T. On delta and nabla Caputo fractional differences and dual identities. Discrete Dyn. Nat. Soc. 2013, 2013, 406910. [CrossRef]

13. Atici, F.M.; Eloe, P.W. Two-point boundary value problems for finite fractional difference equations. J. Differ. Equ. Appl. 2011, 17, 445-456. [CrossRef]

14. Atici, F.M.; Eloe, P.W. A transform method in discrete fractional calculus. Int. J. Differ. Equ. 2007, 2, $165-176$.

15. Atici, F.M.; Eloe, P.W. Initial value problems in discrete fractional calculus. Proc. Amer. Math. Soc. 2009, 137, 981-989. [CrossRef]

16. Agarwal, R.P.; Leanu, D.; Rezapour, S.; Salehi, S. The existence of solutions for some fractional finite difference equations via sum boundary conditions. Adv. Differ. Equ. 2014, 2014, 282. [CrossRef]

17. Goodrich, C.S. On discrete sequential fractional boundary value problems. J. Math. Anal. Appl. 2012, 385, 111-124. [CrossRef] 
18. Goodrich, C.S. On a discrete fractional three-point boundary value problem. J. Differ. Equ. Appl. 2012, 18, 397-415. [CrossRef]

19. Erbe, L.; Goodrich, C.S.; Jia, B.; Peterson, A. Survey of the qualitative properties of fractional difference operators: monotonicity, convexity, and asymptotic behavior of solutions. Adv. Differ. Equ. 2016, 2016, 43. [CrossRef]

20. Chen, Y.; Tang, X. Three difference between a class of discrete fractional and integer order boundary value problems. Commun. Nonlinear Sci. 2014, 19, 4057-4067. [CrossRef]

21. Lv, W.; Feng, J. Nonlinear discrete fractional mixed type sum-difference equation boundary value problems in Banach spaces. Adv. Differ. Equ. 2014, 2014, 184. [CrossRef]

22. Lv, W. Existence of solutions for discrete fractional boundary value problems witha $p$-Laplacian operator. Adv. Differ. Equ. 2012, 2012, 163. [CrossRef]

23. Jia, B.; Erbe, L.; Peterson, A. Two monotonicity results for nabla and delta fractional differences. Arch. Math. 2015, 104, 589-597. [CrossRef]

24. Jia, B.; Erbe, L.; Peterson, A. Convexity for nabla and delta fractional differences. J. Differ. Equ. Appl. 2015, 21, 360-373.

25. Ferreira, R.A.C. Existence and uniqueness of solution to some discrete fractional boundary value problems of order less than one. J. Differ. Equ. Appl. 2013, 19, 712-718. [CrossRef]

26. Ferreira, R.A.C.; Goodrich, C.S. Positive solution for a discrete fractional periodic boundary value problem. Dyn. Contin. Discrete Impuls. Syst. Ser. A Math. Anal. 2012, 19, 545-557.

27. Kang, S.G.; Li, Y.; Chen, H.Q. Positive solutions to boundary value problems of fractional difference equations with nonlocal conditions. Adv. Differ. Equ. 2014, 2014, 7. [CrossRef]

28. Dong, W.; Xu, J.; Regan, D.O. Solutions for a fractional difference boundary value problem. Adv. Differ. Equ. 2013, 2013, 319. [CrossRef]

29. Holm, M. Sum and difference compositions in discrete fractional calculus. Cubo 2011, 13, 153-184. [CrossRef]

30. Sitthiwirattham, T.; Tariboon, J.; Ntouyas, S.K. Existence results for fractional difference equations with three-point fractional sum boundary conditions. Discrete Dyn. Nat. Soc. 2013, 2013, 104276. [CrossRef]

31. Sitthiwirattham, T.; Tariboon, J.; Ntouyas, S.K. Boundary value problems for fractional difference equations with three-point fractional sum boundary conditions. Adv. Differ. Equ. 2013, 2013, 296. [CrossRef]

32. Sitthiwirattham, T. Existence and uniqueness of solutions of sequential nonlinear fractional difference equations with three-point fractional sum boundary conditions. Math. Method. Appl. Sci. 2015, 38, 2809-2815. [CrossRef]

33. Sitthiwirattham, T. Boundary value problem for $p$-Laplacian Caputo fractional difference equations with fractional sum boundary conditions. Math. Method. Appl. Sci. 2016, 39, 1522-1534. [CrossRef]

34. Chasreechai, S.; Kiataramkul, C.; Sitthiwirattham, T. On nonlinear fractional sum-difference equations via fractional sum boundary conditions involving different orders. Math. Probl. Eng. 2015, 2015, 519072 [CrossRef]

35. Reunsumrit, J.; Sitthiwirattham, T. Positive solutions of three-point fractional sum boundary value problem for Caputo fractional difference equations via an argument with a shift. Positivity 2016, 20, 861-876. [CrossRef]

36. Reunsumrit, J.; Sitthiwirattham, T. On positive solutions to fractional sum boundary value problems for nonlinear fractional difference equations. Math. Method. Appl. Sci. 2016, 39, 2737-2751. [CrossRef]

37. Kaewwisetkul, B.; Sitthiwirattham, T. On nonlocal fractional sum-difference boundary value problems for Caputo fractional functional difference equations with delay. Adv. Differ. Equ. 2017, 2017, 219. [CrossRef]

38. Reunsumrit, R.; Sitthiwirattham, T. A New class of four-point fractional sum boundary value problems for nonlinear sequential fractional difference equations involving shift operators. Kragujevac J. Math. 2018, 42, 371-387. [CrossRef]

39. Chasreechai, S.; Sitthiwirattham, T. Existence results of initial value problems for hybrid fractional sum-difference equations. Discrete Dyn. Nat. Soc. 2018, 2018, 5268528. [CrossRef]

40. Chasreechai, S.; Sitthiwirattham, T. On separate fractional sum-difference boundary value problems with n-point fractional sum-difference boundary conditions via arbitrary different fractional orders. Mathematics. 2019, 2019, 471. [CrossRef]

41. Wu, G.C.; Baleanu, D. Discrete fractional logistic map and its chaos. Nonlinear Dyn. 2014, 75, $283-287$. [CrossRef] 
42. Wu, G.C.; Baleanu, D. Chaos synchronization of the discrete fractional logistic map. Signal Process. 2014, 102, 96-99. [CrossRef]

43. Wu, G.C.; Baleanu, D.; Xie, H.P.; Chen, F.L. Chaos synchronization of fractional chaotic maps based on stability results. Phys. A 2016, 460, 374-383. [CrossRef]

44. Chen, C.; Bohner, M.; Jia, B. Ulam-Hyers stability of Caputo fractional difference equations. Math. Meth. Appl. Sci. 2019, 42, 7461-7470. [CrossRef]

45. Wu, G.C.; Baleanu, D.; Zeng, S.D. Finite-time stability of discrete fractional delay systems: Gronwall inequality and stability criterion. Commun. Nonlinear Sci. Numer. Simulat. 2018, 57, 299-308. [CrossRef]

46. Baleanu, D.; Wu, G.C.; Bai, Y.R.; Chen, F.L. Stability analysis of Caputo-like discrete fractional systems. Commun. Nonlinear Sci. Numer. Simulat. 2017, 48, 520-530. [CrossRef]

47. Chen, F.; Zhou, Y. Existence and Ulam Stability of Solutions for Discrete Fractional Boundary Value Problem. Discrete Dyn. Nat. Soc. 2013, 2013, 459161. [CrossRef]

48. Cermák, J.; Gőrigyori, I.; Nechvátal, L. On explicit stability conditions for a linear fractional difference system. Fract. Calc. Appl. Anal. 2015, 18, 651-672. [CrossRef]

49. Lizama, C. The Poisson distribution, abstract fractional difference equations, and stability. Proc. Am. Math. Soc. 2017, 145, 3809-3827. [CrossRef]

50. Cermák, J.; Kisela, T.; Nechvátal, L. Stability and asymptotic properties of a linear fractional difference equation. Adv. Differ. Equ. 2012, 2012, 122. [CrossRef]

51. Dassios, I.K. Stability and Robustness of Singular Systems of Fractional Nabla Difference Equations. Circuits Syst. Signal Process 2017, 36, 49-64. [CrossRef]

52. Wu, G.C.; Baleanu, D. Stability analysis of impulsive fractional difference equations. Fract. Calc. Appl. Anal. 2018, 21, 354-375. [CrossRef]

53. Wu, G.C.; Baleanu, D.; Huang, L.L. Novel Mittag-Leffler stability of linear fractional delay difference equations with impulse. Appl. Math. Lett. 2018, 82, 71-78. [CrossRef]

54. Wang, J.; Lv, L.; Zhou, Y. Ulam stability and data dependence for fractional differential equations with Caputo derivative. Electron. J. Qual. Theory Differ. Equ. 2011, 63, 1-10. [CrossRef]

(C) 2020 by the authors. Licensee MDPI, Basel, Switzerland. This article is an open access article distributed under the terms and conditions of the Creative Commons Attribution (CC BY) license (http:/ / creativecommons.org/licenses/by/4.0/). 